Honam Mathematical J. 34 (2012), No. 3, pp. 327-340

http://dx.doi.org/10.5831/HMJ.2012.34.3.327

\title{
$q$-EXTENSION OF A GENERALIZATION OF GOTTLIEB POLYNOMIALS IN THREE VARIABLES
}

\author{
JunESANG CHOI
}

\begin{abstract}
Gottlieb polynomials were introduced and investigated in 1938, and then have been cited in several articles. Very recently Khan and Akhlaq introduced and investigated Gottlieb polynomials in two and three variables to give their generating functions. Subsequently, Khan and Asif investigated the generating functions for the $q$-analogue of Gottlieb polynomials. Very recently, Choi defined a $q$-extension of the generalized two variable Gottlieb polynomials $\varphi_{n}^{2}(\cdot)$ and presented their several generating functions. Also, by modifying Khan and Akhlaq's method, Choi presented a generalization of the Gottlieb polynomials in $m$ variables to give two generating functions of the generalized Gottlieb polynomials $\varphi_{n}^{m}(\cdot)$. Here, in the sequel of the above results for their possible general $q$-extensions in several variables, again, we aim at trying to define a $q$-extension of the generalized three variable Gottlieb polynomials $\varphi_{n}^{3}(\cdot)$ and present their several generating functions.
\end{abstract}

\section{Introduction and Preliminaries}

Generating functions play an important role in the investigation of various useful properties of the sequences which they generate. They are used in finding certain properties and formulas for numbers and polynomials in a wide variety of research subjects, indeed, in modern combinatorics. For a systematic introduction to, and several interesting (and useful) applications of the various methods of obtaining linear, bilinear, bilateral or mixed multilateral generating functions for a fairly wide variety of sequences of special functions (and polynomials) in one,

Received April 24, 2012. Accepted May 22, 2012.

2000 Mathematics Subject Classification. Primary 33C65, 33C99; Secondary $33 \mathrm{C} 05,33 \mathrm{C} 20$.

Key words and phrases. Pochhammer symbol; Generating functions; Generalized hypergeometric function ${ }_{p} F_{q}$; (Generalized) Gottlieb polynomials; Lauricella series; a $q$-analogue of Gottlieb polynomials; $q$-shifted factorial; $q$-binomial theorem; Basic $(q-)$ hypergeometric series. 
two and more variables, among much abundant literature, we refer to the extensive work by Srivastava and Manocha [11]. While concerning some orthogonal polynomials on a finite or enumerable set of points, Gottlieb [5] developed the following interesting polynomials (see also [2]; [6]; [7]; [9, p. 303]; [11, pp. 185-186]):

$$
\begin{aligned}
\varphi_{n}(x ; \lambda): & =e^{-n \lambda} \sum_{k=0}^{n}\left(\begin{array}{l}
n \\
k
\end{array}\right)\left(\begin{array}{l}
x \\
k
\end{array}\right)\left(1-e^{\lambda}\right)^{k} \\
& =e^{-n \lambda}{ }_{2} F_{1}\left(-n,-x ; 1 ; 1-e^{\lambda}\right),
\end{aligned}
$$

where ${ }_{2} F_{1}$ denotes Gauss's hypergeometric series whose natural generalization of an arbitrary number of $p$ numerator and $q$ denominator parameters ( $p, q \in \mathbb{N}_{0}:=\mathbb{N} \cup\{0\}$, and $\mathbb{N}$ the set of positive integers) is called and denoted by the generalized hypergeometric series ${ }_{p} F_{q}$ defined by

$$
\begin{aligned}
{ }_{p} F_{q}\left[\begin{array}{c}
\alpha_{1}, \ldots, \alpha_{p} ; z \\
\beta_{1}, \ldots, \beta_{q} ; z
\end{array}\right] & =\sum_{n=0}^{\infty} \frac{\left(\alpha_{1}\right)_{n} \cdots\left(\alpha_{p}\right)_{n}}{\left(\beta_{1}\right)_{n} \cdots\left(\beta_{q}\right)_{n}} \frac{z^{n}}{n !} \\
& ={ }_{p} F_{q}\left(\alpha_{1}, \ldots, \alpha_{p} ; \beta_{1}, \ldots, \beta_{q} ; z\right) .
\end{aligned}
$$

Here $(\lambda)_{n}$ is the Pochhammer symbol defined (for $\lambda \in \mathbb{C}$ ) by

$$
\begin{aligned}
(\lambda)_{n}: & = \begin{cases}1 & (n=0) \\
\lambda(\lambda+1) \ldots(\lambda+n-1) & (n \in \mathbb{N})\end{cases} \\
& =\frac{\Gamma(\lambda+n)}{\Gamma(\lambda)} \quad\left(\lambda \in \mathbb{C} \backslash \mathbb{Z}_{0}^{-}\right)
\end{aligned}
$$

and $\mathbb{C}$ and $\mathbb{Z}_{0}^{-}$denotes the set of nonpositive integers and the set of complex numbers, respectively, and $\Gamma(\lambda)$ is the familiar Gamma function.

Gottlieb [5] presented many interesting identities for his polynomials $\varphi_{n}(x ; \lambda)$, which is denoted by $l_{n}(x)$ in [5], including the following two generating functions (see also [6]; [7]; [9, p. 303]; [11, pp. 185-186]):

$$
\sum_{n=0}^{\infty} \varphi_{n}(x ; \lambda) t^{n}=(1-t)^{x}\left(1-t e^{-\lambda}\right)^{-x-1} \quad(|t|<1) ;
$$

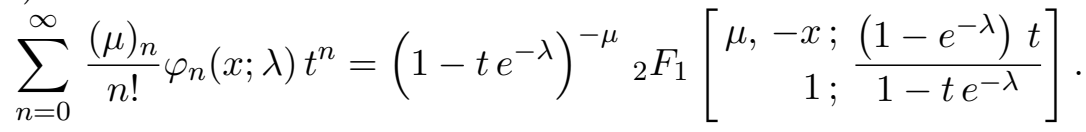

Recently Khan and Akhlaq [6] introduced and investigated Gottlieb polynomials in two and three variables to give their generating functions. 
Subsequently, Khan and Asif [7] investigated the generating functions for a $q$-analogue of Gottlieb polynomials. Very recently, Choi [3] defined a q-extension of Also, by modifying Khan and Akhlaq's method [6], Choi [2] presented a generalization of the Gottlieb polynomials in $m$ variables to give two generating functions of the generalized Gottlieb polynomials $\varphi_{n}^{m}(\cdot)$. Basic (or $q$-) hypergeometric series are useful in a wide variety of fields including, for example, theory of partitions, number theory, combinatorial analysis, finite vector space, Lie theory, particle physics, non-linear electric circuit theory, mechanical engineering, theory of heat conduction, quantum mechanics, cosmology, and statistics (see [10, 346351 ] and also see the cited references therein). Here, in the sequel of the above results for their possible general $q$-extensions in several variables, again, we aim at trying to define a $q$-extension of the generalized three variable Gottlieb polynomials $\varphi_{n}^{3}(\cdot)$ and present their several generating functions.

For our purpose we recall here the following definitions and notations in the $q$-theory (see, for example, [4]). The $q$-shifted factorial $(a ; q)_{n}$ is defined by

$$
(a ; q)_{n}:= \begin{cases}1 & (n=0) \\ \prod_{k=0}^{n-1}\left(1-a q^{k}\right) & (n \in \mathbb{N}),\end{cases}
$$

where $a, q \in \mathbb{C}$ and it is assumed that $a \neq q^{-m}\left(m \in \mathbb{N}_{0}\right)$. It is noted that some other notations that have been used in the literature for the product $(a ; q)_{n}$ in $(1.6)$ are $(a)_{q, n},[a]_{n}$, and even $(a)_{n}$ when the Pochhammer symbol (1.3) is not used and the base $q$ is understood.

The $q$-shifted factorial for negative subscript is defined by

$$
(a ; q)_{-n}:=\frac{1}{\left(1-a q^{-1}\right)\left(1-a q^{-2}\right) \cdots\left(1-a q^{-n}\right)} \quad\left(n \in \mathbb{N}_{0}\right),
$$

which yields

$$
(a ; q)_{-n}=\frac{1}{\left(a q^{-n} ; q\right)_{n}}=\frac{(-q / a)^{n} q^{\left(\begin{array}{c}
n \\
2
\end{array}\right)}}{(q / a ; q)_{n}} \quad\left(n \in \mathbb{N}_{0}\right) .
$$

We also write

$$
(a ; q)_{\infty}:=\prod_{k=0}^{\infty}\left(1-a q^{k}\right) \quad(a, q \in \mathbb{C} ;|q|<1) .
$$


It is noted that, when $a \neq 0$ and $|q| \geqq 1$, the infinite product in (1.9) diverges. So, whenever $(a ; q)_{\infty}$ is involved in a given formula, the constraint $|q|<1$ will be tacitly assumed.

It follows from (1.6), (1.7) and (1.9) that

$$
(a ; q)_{n}=\frac{(a ; q)_{\infty}}{\left(a q^{n} ; q\right)_{\infty}} \quad(n \in \mathbb{Z}),
$$

$\mathbb{Z}$ being the set of integers, which can be extended to $n=\alpha \in \mathbb{C}$ as follows:

$$
(a ; q)_{\alpha}=\frac{(a ; q)_{\infty}}{\left(a q^{\alpha} ; q\right)_{\infty}} \quad(\alpha \in \mathbb{C} ;|q|<1),
$$

where the principal value of $q^{\alpha}$ is taken.

Two easily-verified required identities are given:

$$
\left(q^{-n} ; q\right)_{k}=\frac{(q ; q)_{n}}{(q ; q)_{n-k}}(-1)^{k} q^{\left(\begin{array}{c}
k \\
2
\end{array}\right)-n k} \quad(n, k \in \mathbb{Z})
$$

and

$$
(a ; q)_{n+k}=(a ; q)_{n}\left(a q^{n} ; q\right)_{k} \quad(n, k \in \mathbb{Z}) .
$$

The notation $[z]_{q}$ is defined by

$$
[z]_{q}:=\frac{1-q^{z}}{1-q}=\frac{q^{z}-1}{q-1} \quad\left(z \in \mathbb{C} ; q \in \mathbb{C} \backslash\{1\} ; q^{z} \neq 1\right) .
$$

A special case of (1.14) when $z \in \mathbb{N}$ is

$$
[n]_{q}=\frac{q^{n}-1}{q-1}=1+q+\cdots+q^{n-1} \quad(n \in \mathbb{N}),
$$

which is called the $q$-analogue (or q-extension) of $n \in \mathbb{N}$, since

$$
\lim _{q \rightarrow 1}[n]_{q}=\lim _{q \rightarrow 1}\left(1+q+\cdots+q^{n-1}\right)=n .
$$

The $q$-analogue of $n$ ! is then defined by

$$
[n]_{q} !:= \begin{cases}1 & \text { if } n=0, \\ {[n]_{q}[n-1]_{q} \cdots[2]_{q}[1]_{q}} & \text { if } n \in \mathbb{N},\end{cases}
$$

from which the q-binomial coefficient (or the Gaussian polynomial) analogous to $\left(\begin{array}{l}n \\ k\end{array}\right)$ is defined by

$$
\left[\begin{array}{l}
n \\
k
\end{array}\right]_{q}:=\frac{[n]_{q} !}{[n-k]_{q} ![k]_{q} !} \quad\left(n, k \in \mathbb{N}_{0} ; 0 \leqq k \leqq n\right) .
$$

It is easily seen from (1.6) and (1.17) that

$$
(q ; q)_{n}=(1-q)^{n}[n]_{q} ! \quad\left(n \in \mathbb{N}_{0}\right) .
$$


The $q$-binomial coefficient in (1.17) can be generalized as follows:

$$
\left[\begin{array}{l}
\alpha \\
k
\end{array}\right]_{q}:=\frac{[\alpha]_{q ; k}}{[k]_{q} !} \quad\left(\alpha \in \mathbb{C} ; k \in \mathbb{N}_{0}\right),
$$

where $[\alpha]_{q ; k}$ is defined by

$$
[\alpha]_{q ; k}:=[\alpha]_{q}[\alpha-1]_{q} \cdots[\alpha-k+1]_{q} \quad\left(\alpha \in \mathbb{C} ; k \in \mathbb{N}_{0}\right) .
$$

The generalized $q$-binomial coefficient in (1.19) can be expressed in the following form:

$$
\left[\begin{array}{l}
\alpha \\
k
\end{array}\right]_{q}=\frac{\left(q^{-\alpha} ; q\right)_{k}}{(q ; q)_{k}}\left(-q^{\alpha}\right)^{k} q^{-\left(\begin{array}{c}
k \\
2
\end{array}\right)} \quad\left(\alpha \in \mathbb{C} ; k \in \mathbb{N}_{0}\right) .
$$

The following notations are also frequently used:

$$
\left(a_{1}, a_{2}, \cdots, a_{m} ; q\right)_{n}:=\left(a_{1} ; q\right)_{n}\left(a_{2} ; q\right)_{n} \cdots\left(a_{m} ; q\right)_{n}
$$

and

$$
\left(a_{1}, a_{2}, \cdots, a_{m} ; q\right)_{\infty}:=\left(a_{1} ; q\right)_{\infty}\left(a_{2} ; q\right)_{\infty} \cdots\left(a_{m} ; q\right)_{\infty} .
$$

In order to introduce $q$-binomial theorem, we begin by recalling the well-known Ramanujan's ${ }_{1} \Psi_{1}$-sum:

$$
\begin{gathered}
{ }_{1} \Psi_{1}(a ; b ; q, z):=\sum_{k=-\infty}^{\infty} \frac{(a ; q)_{k}}{(b ; q)_{k}} z^{k}=\frac{(a z ; q)_{\infty}\left(\frac{q}{a z} ; q\right)_{\infty}(q ; q)_{\infty}\left(\frac{b}{a} ; q\right)_{\infty}}{(z ; q)_{\infty}\left(\frac{b}{a z} ; q\right)_{\infty}(b ; q)_{\infty}\left(\frac{q}{a} ; q\right)_{\infty}} \\
(|q|<1 ;|a|>|q| ;|b|<1 ;|b / a|<|z|<1) .
\end{gathered}
$$

A special case of (1.24) when $b=q$ yields the $q$-binomial theorem:

$$
{ }_{1} \Phi_{0}(a ;-; q, z):=\sum_{k=0}^{\infty} \frac{(a ; q)_{k}}{(q ; q)_{k}} z^{k}=\frac{(a z ; q)_{\infty}}{(z ; q)_{\infty}} \quad(|q|<1 ;|z|<1) .
$$

Two special cases of (1.25) when $a=0$ and when $z$ is replaced by $z a^{-1}$ and $a \rightarrow \infty$ yield Euler's formulas:

$$
\sum_{k=0}^{\infty} \frac{z^{k}}{(q ; q)_{k}}=\frac{1}{(z ; q)_{\infty}} \quad(|q|<1 ;|z|<1)
$$

and

$$
\sum_{k=0}^{\infty} \frac{(-1)^{k} q^{\left(\begin{array}{c}
k \\
2
\end{array}\right)}}{(q ; q)_{k}} z^{k}=(z ; q)_{\infty} \quad(|q|<1 ;|z|<1),
$$

respectively. 
It is observed that

$$
\begin{gathered}
\lim _{q \downarrow 1} \frac{\left(q^{a} z ; q\right)_{\infty}}{(z ; q)_{\infty}}=\lim _{q \downarrow 1}{ }_{1} \Phi_{0}\left(q^{a} ;-; q, z\right)={ }_{1} F_{0}(a ;-; z)=(1-z)^{-a} \\
(|z|<1 ; a \in \mathbb{C}),
\end{gathered}
$$

which, by the principle of analytic continuation, holds true for $z \in \mathbb{C}$ cut along the positive real axis from 1 to $\infty$, with $(1-z)^{-a}$ positive when $z$ is real and less than 1 .

A $q$-analogue of the classical exponential function $e^{z}$ is defined by

$$
e_{q}(z):=\sum_{k=0}^{\infty} \frac{z^{k}}{[k]_{q} !}
$$

and another $q$-analogue of the classical exponential function $e^{z}$ is defined by

$$
E_{q}(z):=\sum_{k=0}^{\infty} q^{k(k-1) / 2} \frac{z^{k}}{[k]_{q} !} .
$$

It is easily seen by applying (1.29) and (1.30) that

$$
e_{q}(z) E_{q}(-z)=1 .
$$

We conclude this section by giving a widely-investigated generalization ${ }_{r} \Phi_{s}$ of the function ${ }_{1} \Phi_{0}(a ;-; q, z)$ in (1.25), which is defined by

$$
\begin{array}{r}
{ }_{r} \Phi_{s}\left[\begin{array}{c}
a_{1}, \cdots, a_{r} ; \\
b_{1}, \cdots, b_{s} ;
\end{array}\right]={ }_{r} \Phi_{s}\left(a_{1}, \cdots, a_{r} ; b_{1}, \cdots, b_{s} ; q, z\right) \\
\quad:=\sum_{k=0}^{\infty}(-1)^{(1-r+s) k} q^{(1-r+s)\left(\begin{array}{c}
k \\
2
\end{array}\right)} \frac{\left(a_{1} ; q\right)_{k} \cdots\left(a_{r} ; q\right)_{k}}{\left(b_{1} ; q\right)_{k} \cdots\left(b_{s} ; q\right)_{k}} \frac{z^{k}}{(q ; q)_{k}},
\end{array}
$$

provided that the generalized basic (or q-) hypergeometric series in (1.32) converges.

\section{Definitions of $q$-Extensions of Gottlieb and three variable Gottlieb polynomials}

We begin by recalling the definition of a several variable analogue of the Gottlieb polynomials $\varphi_{n}(x ; \lambda)$ and one of their generating functions in $[2]$. 
Definition 1. An extension of the Gottlieb polynomials $\varphi_{n}(x ; \lambda)$ in $m$ variables is defined by

$$
\begin{aligned}
& \varphi_{n}^{m}\left(x_{1}, x_{2}, \ldots, x_{m} ; \lambda_{1}, \lambda_{2}, \ldots, \lambda_{m}\right) \\
& =\exp \left(-n \sigma_{m}\right) \sum_{r_{1}=0}^{n} \sum_{r_{2}=0}^{n-r_{1}} \sum_{r_{3}=0}^{n-r_{1}-r_{2}} \cdots \sum_{r_{m}=0}^{n-r_{1}-r_{2}-\cdots-r_{m-1}} \\
& \cdot \frac{(-n)_{\delta_{m}} \cdot \prod_{j=1}^{m}\left(-x_{j}\right)_{r_{j}} \cdot \prod_{j=1}^{m}\left(1-e^{\lambda_{j}}\right)^{r_{j}}}{\delta_{m} ! \cdot \prod_{j=1}^{m} r_{j} !} \quad(n, m \in \mathbb{N}),
\end{aligned}
$$

where, for convenience,

$$
\sigma_{m}:=\sum_{j=1}^{m} \lambda_{j} \quad \text { and } \quad \delta_{m}:=\sum_{j=1}^{m} r_{j} .
$$

It is noted that the special case $m=1$ of $(2.1)$ reduces immediately to the second one of the Gottlieb polynomials $\varphi_{n}(x ; \lambda)$ in $(1.1)$ and the cases of (2.1) when $m=2$ and $m=3$ correspond with those in $[6,7]$.

The following generating function for $\varphi_{n}^{m}\left(x_{1}, x_{2}, \ldots, x_{m} ; \lambda_{1}, \lambda_{2}, \ldots\right.$, $\lambda_{m}$ ) holds true:

$$
\begin{aligned}
& \sum_{n=0}^{\infty}(\mu)_{n} \varphi_{n}^{m}\left(x_{1}, x_{2}, \ldots, x_{m} ; \lambda_{1}, \lambda_{2}, \ldots, \lambda_{m}\right) \frac{t^{n}}{n !}=\left(1-t e^{-\sigma_{m}}\right)^{-\mu} \\
& \cdot F_{D}^{(m)}\left[\mu,-x_{1}, \ldots,-x_{m} ; 1 ; \frac{t\left(e^{\lambda_{1}}-1\right) e^{-\sigma_{m}}}{1-t e^{-\sigma_{m}}}, \ldots, \frac{t\left(e^{\lambda_{m}}-1\right) e^{-\sigma_{m}}}{1-t e^{-\sigma_{m}}}\right],
\end{aligned}
$$

where $F_{D}^{(m)}[\cdot]$ denotes one of the Lauricella series in $m$ variables (see [10, p. 33, Eq. (4)]; see also [8]) defined by

$$
\begin{aligned}
& F_{D}^{(m)}\left[a, b_{1}, \ldots, b_{m} ; c ; x_{1}, \ldots, x_{m}\right] \\
& =\sum_{\substack{r_{1}=0, \ldots, r_{m}=0 \\
(\max }}^{\infty} \frac{\left.\left.(a)_{\delta_{m}}\left(b_{1}\right)_{r_{1}} \cdots\left(b_{m}\right)_{r_{m}}|, \ldots,| x_{m} \mid\right\}<1\right)}{(c)_{\delta_{m}}} \frac{x_{1}^{r_{1}}}{r_{1} !} \cdots \frac{x_{m}^{r_{m}}}{r_{m} !}
\end{aligned}
$$

and $\sigma_{m}, \delta_{m}$ are given in (2.2).

Khan and Asif [7] defined a $q$-analogue of Gottlieb polynomials in (1.1) given below. 
Definition 2. A $q$-extension of the Gottlieb polynomials $\varphi_{n}(x ; \lambda)$ is given as follows:

$$
\varphi_{n ; q}(x ; \lambda)=\left\{E_{q}(-\lambda)\right\}^{n} \sum_{k=0}^{n}\left[\begin{array}{l}
n \\
k
\end{array}\right]_{q}\left[\begin{array}{l}
x \\
k
\end{array}\right]_{q} \cdot q^{k(k-1)-x k} \cdot\left(1-e_{q}(\lambda)\right)^{k}
$$

By using (1.12), (1.17) and (1.19), Khan and Asif [7] gave another expression of the $q$-extension of the Gottlieb polynomials $\varphi_{n}(x ; \lambda)$ in $(2.5)$ :

$$
\varphi_{n ; q}(x ; \lambda)=\left\{E_{q}(-\lambda)\right\}_{2}^{n} \Phi_{1}\left[\begin{array}{rr}
q^{-n}, & \left.q^{-x} ; q, q^{n}\left(1-e_{q}(\lambda)\right)\right] . \\
& q ;
\end{array}\right.
$$

Khan and Asif [7] used some of the identities given in Section 1 to present the following three generating functions for $q$-Gottlieb polynomials in (2.5):

$$
\sum_{n=0}^{\infty} \varphi_{n ; q}(x ; \lambda) t^{n}=\left(1-t E_{q}(-\lambda)\right)^{-1}{ }_{1} \Phi_{1}\left[\begin{array}{r}
q^{-x} \\
q t E_{q}(-\lambda) ;
\end{array} q,-\left(1-E_{q}(-\lambda)\right) t\right] ;
$$

$$
\sum_{n=0}^{\infty} \varphi_{n ; q}(x ; \lambda) \frac{t^{n}}{(q ; q)_{n}}=e_{q}\left(t E_{q}(-\lambda)\right)_{1} \Phi_{1}\left[\begin{array}{r}
\left.q^{-x} ; q,-\left(1-E_{q}(-\lambda)\right) t\right] \\
q ;
\end{array}\right.
$$

$$
\begin{aligned}
& \sum_{n=0}^{\infty} \frac{\left(q^{c} ; q\right)_{n}}{(q ; q)_{n}} \varphi_{n ; q}(x ; \lambda) t^{n} \\
& \quad=\frac{\left(t q^{c} E_{q}(-\lambda) ; q\right)_{\infty}}{\left(t E_{q}(-\lambda) ; q\right)_{\infty}}{ }_{2} \Phi_{2}\left[\begin{array}{rr}
q^{c}, & q^{-x} ; \\
q, & t q^{c} E_{q}(-\lambda) ;
\end{array},-\left(1-E_{q}(-\lambda)\right) t\right]
\end{aligned}
$$

We find from Definition 1 that the three variable Gottlieb polynomials $\varphi_{n}^{3}\left(x_{1}, x_{2}, x_{3} ; \lambda_{1}, \lambda_{2}, \lambda_{3}\right)$ is given by

$$
\begin{aligned}
& \varphi_{n}^{3}\left(x_{1}, x_{2}, x_{3} ; \lambda_{1}, \lambda_{2}, \lambda_{3}\right)=\exp \left(-n\left(\lambda_{1}+\lambda_{2}+\lambda_{3}\right)\right) \\
& \cdot \sum_{r_{1}=0}^{n} \sum_{r_{2}=0}^{n-r_{1}} \sum_{r_{3}=0}^{n-r_{1}-r_{2}} \frac{(-n)_{r_{1}+r_{2}+r_{3}}\left(-x_{1}\right)_{r_{1}}\left(-x_{2}\right)_{r_{2}}\left(-x_{3}\right)_{r_{3}}}{r_{1} ! r_{2} ! r_{3} !\left(r_{1}+r_{2}+r_{3}\right) !} \\
& \quad \cdot\left(1-e^{\lambda_{1}}\right)^{r_{1}}\left(1-e^{\lambda_{2}}\right)^{r_{2}}\left(1-e^{\lambda_{3}}\right)^{r_{3}} \quad(n \in \mathbb{N}),
\end{aligned}
$$


which is easily rewritten as follows:

$$
\begin{gathered}
\varphi_{n}^{3}\left(x_{1}, x_{2}, x_{3} ; \lambda_{1}, \lambda_{2}, \lambda_{3}\right)=\exp \left(-n\left(\lambda_{1}+\lambda_{2}+\lambda_{3}\right)\right) \\
\cdot \sum_{r_{1}=0}^{n} \sum_{r_{2}=0}^{n-r_{1}} \sum_{r_{3}=0}^{n-r_{1}-r_{2}}\left(\begin{array}{c}
n \\
r_{1}+r_{2}+r_{3}
\end{array}\right)\left(\begin{array}{l}
x_{1} \\
r_{1}
\end{array}\right)\left(\begin{array}{l}
x_{2} \\
r_{2}
\end{array}\right)\left(\begin{array}{l}
x_{3} \\
r_{3}
\end{array}\right) \\
\cdot\left(1-e^{\lambda_{1}}\right)^{r_{1}}\left(1-e^{\lambda_{2}}\right)^{r_{2}}\left(1-e^{\lambda_{3}}\right)^{r_{3}} \quad(n \in \mathbb{N}) .
\end{gathered}
$$

We define a $q$-extension of $\varphi_{n}^{3}\left(x_{1}, x_{2}, x_{3} ; \lambda_{1}, \lambda_{2}, \lambda_{3}\right)$.

Definition 3. A $q$-extension of the three variable Gottlieb polynomials $\varphi_{n}^{3}\left(x_{1}, x_{2}, x_{3} ; \lambda_{1}, \lambda_{2}, \lambda_{3}\right)$ is defined as follows:

$$
\begin{aligned}
& \varphi_{n ; q}^{3}\left(x_{1}, x_{2}, x_{3} ; \lambda_{1}, \lambda_{2}, \lambda_{3}\right):=\left\{E_{q}\left(-\lambda_{1}\right) E_{q}\left(-\lambda_{2}\right) E_{q}\left(-\lambda_{3}\right)\right\}^{n} \\
& \quad \cdot \sum_{r_{1}=0}^{n} \sum_{r_{2}=0}^{n-r_{1}} \sum_{r_{3}=0}^{n-r_{1}-r_{2}}\left[\begin{array}{c}
n \\
r_{1}+r_{2}+r_{3}
\end{array}\right]_{q}\left[\begin{array}{l}
x_{1} \\
r_{1}
\end{array}\right]_{q}\left[\begin{array}{l}
x_{2} \\
r_{2}
\end{array}\right]_{q}\left[\begin{array}{l}
x_{3} \\
r_{3}
\end{array}\right]_{q} \\
& \cdot q^{\left(\begin{array}{c}
r_{1} \\
2
\end{array}\right)+\left(\begin{array}{c}
r_{2} \\
2
\end{array}\right)+\left(\begin{array}{c}
r_{3} \\
2
\end{array}\right)-x_{1} r_{1}-x_{2} r_{2}-x_{3} r_{3}} \\
& \quad \cdot\left(1-e_{q}\left(\lambda_{1}\right)\right)^{r_{1}}\left(1-e_{q}\left(\lambda_{2}\right)\right)^{r_{2}}\left(1-e_{q}\left(\lambda_{3}\right)\right)^{r_{3}}
\end{aligned}
$$

which, upon making use of Equations (1.12), (1.17) and (1.21), can be expressed in the following form:

$$
\begin{aligned}
& \stackrel{(2.13)}{\varphi_{n ; q}^{3}}\left(x_{1}, x_{2}, x_{3} ; \lambda_{1}, \lambda_{2}, \lambda_{3}\right)=\left\{E_{q}\left(-\lambda_{1}\right) E_{q}\left(-\lambda_{2}\right) E_{q}\left(-\lambda_{3}\right)\right\}^{n} \\
& \quad \cdot \sum_{r_{1}=0}^{n} \sum_{r_{2}=0}^{n-r_{1}} \sum_{r_{3}=0}^{n-r_{1}-r_{2}} \frac{\left.q^{-\left(r_{1}+r_{2}+r_{3}\right)}\left(q^{-n} ; q\right)_{r_{1}+r_{2}+r_{3}}\left(q^{-x_{1}} ; q\right)_{r_{1}}\left(q^{-x_{2}} ; q\right)_{r_{2}}\left(q^{-x_{3}} ; q\right)\right)_{r_{3}}}{(q ; q)_{r_{1}+r_{2}+r_{3}}(q ; q)_{r_{1}}(q ; q)_{r_{2}}(q ; q)_{r_{3}}} \\
& \quad \cdot\left\{q^{n}\left(1-e_{q}\left(\lambda_{1}\right)\right)\right\}^{r_{1}}\left\{q^{n}\left(1-e_{q}\left(\lambda_{2}\right)\right)\right\}^{r_{2}}\left\{q^{n}\left(1-e_{q}\left(\lambda_{3}\right)\right)\right\}^{r_{3}} .
\end{aligned}
$$

3. Generating functions for $\varphi_{n ; q}^{3}\left(x_{1}, x_{2}, x_{3} ; \lambda_{1}, \lambda_{2}, \lambda_{3}\right)$

Here, we aim at presenting three generating functions for $\varphi_{n ; q}^{3}\left(x_{1}, x_{2}\right.$, ,$\left.x_{3} ; \lambda_{1}, \lambda_{2}, \lambda_{3}\right)$ asserted by Theorem below. 
Theorem. Each of the following generating functions for the $\varphi_{n ; q}^{3}\left(x_{1}, x_{2}, x_{3} ; \lambda_{1}, \lambda_{2}, \lambda_{3}\right)$ holds true:

$$
\begin{gathered}
\sum_{n=0}^{\infty} \varphi_{n ; q}^{3}\left(x_{1}, x_{2}, x_{3} ; \lambda_{1}, \lambda_{2}, \lambda_{3}\right) t^{n}=\frac{\left(q t E_{q}\left(-\lambda_{1}\right) E_{q}\left(-\lambda_{2}\right) E_{q}\left(-\lambda_{3}\right) ; q\right)_{\infty}}{\left(t E_{q}\left(-\lambda_{1}\right) E_{q}\left(-\lambda_{2}\right) E_{q}\left(-\lambda_{3}\right) ; q\right)_{\infty}} \\
\cdot \Phi_{1: 0 ; 0 ; 0}^{0: 1 ; 1 ; 1}\left(\begin{array}{c}
--: \\
{\left[q t E_{q}\left(-\lambda_{1}\right) E_{q}\left(-\lambda_{2}\right) E_{q}\left(-\lambda_{3}\right): 1,1,1\right]:} \\
{\left[q^{-x_{1}}: 1\right] ; \quad\left[q^{-x_{2}}: 1\right] ; \quad\left[q^{-x_{3}}: 1\right] ;} \\
--; \\
--;
\end{array} \quad--;\right. \\
q ; t E_{q}\left(-\lambda_{2}\right) E_{q}\left(-\lambda_{3}\right)\left(1-E_{q}\left(-\lambda_{1}\right)\right), t E_{q}\left(-\lambda_{1}\right) E_{q}\left(-\lambda_{3}\right)\left(1-E_{q}\left(-\lambda_{2}\right)\right), \\
\left.t E_{q}\left(-\lambda_{1}\right) E_{q}\left(-\lambda_{2}\right)\left(1-E_{q}\left(-\lambda_{3}\right)\right)\right) ;
\end{gathered}
$$

$$
\begin{aligned}
& \sum_{n=0}^{\infty} \varphi_{n ; q}^{3}\left(x_{1}, x_{2}, x_{3} ; \lambda_{1}, \lambda_{2}, \lambda_{3}\right) \frac{t^{n}}{(q ; q)_{n}}=\frac{1}{\left(t E_{q}\left(-\lambda_{1}\right) E_{q}\left(-\lambda_{2}\right) E_{q}\left(-\lambda_{3}\right) ; q\right)_{\infty}} \\
& \cdot \Phi_{1: 0 ; 0 ; 0}^{0: 1 ; 1 ; 1}\left(\begin{array}{rrrr}
--: & {\left[q^{-x_{1}}: 1\right] ;} & {\left[q^{-x_{2}}: 1\right] ;} & {\left[q^{-x_{3}}: 1\right] ;} \\
{[q: 1,1,1]:} & --; & --; & --;
\end{array}\right. \\
& q ; t E_{q}\left(-\lambda_{2}\right) E_{q}\left(-\lambda_{3}\right)\left(1-E_{q}\left(-\lambda_{1}\right)\right), t E_{q}\left(-\lambda_{1}\right) E_{q}\left(-\lambda_{3}\right)\left(1-E_{q}\left(-\lambda_{2}\right)\right) \text {, } \\
& \left.t E_{q}\left(-\lambda_{1}\right) E_{q}\left(-\lambda_{2}\right)\left(1-E_{q}\left(-\lambda_{3}\right)\right)\right) \text {; }
\end{aligned}
$$

$$
\begin{aligned}
& \sum_{n=0}^{\infty} \frac{\left(q^{c} ; q\right)_{n}}{(q ; q)_{n}} \varphi_{n ; q}^{3}\left(x_{1}, x_{2}, x_{3} ; \lambda_{1}, \lambda_{2}, \lambda_{3}\right) t^{n} \\
& =\frac{\left(q^{c} t E_{q}\left(-\lambda_{1}\right) E_{q}\left(-\lambda_{2}\right) E_{q}\left(-\lambda_{3}\right) ; q\right)_{\infty}}{\left(t E_{q}\left(-\lambda_{1}\right) E_{q}\left(-\lambda_{2}\right) E_{q}\left(-\lambda_{3}\right) ; q\right)_{\infty}} \\
& \text {. } \Phi_{2: 0 ; 0 ; 0}^{1: 1 ; 1 ; 1}\left(\left[q, q^{c} t E_{q}\left(-\lambda_{1}\right) E_{q}\left(-\lambda_{2}\right) E_{q}\left(-\lambda_{3}\right): 1,1,1\right]:\right. \\
& {\left[q^{-x_{1}}: 1\right] ; \quad\left[q^{-x_{2}}: 1\right] ; \quad\left[q^{-x_{3}}: 1\right] ;} \\
& q ; t E_{q}\left(-\lambda_{2}\right) E_{q}\left(-\lambda_{3}\right)\left(1-E_{q}\left(-\lambda_{1}\right)\right), t E_{q}\left(-\lambda_{1}\right) E_{q}\left(-\lambda_{3}\right)\left(1-E_{q}\left(-\lambda_{2}\right)\right), \\
& \left.t E_{q}\left(-\lambda_{1}\right) E_{q}\left(-\lambda_{2}\right)\left(1-E_{q}\left(-\lambda_{3}\right)\right)\right) \text {, }
\end{aligned}
$$




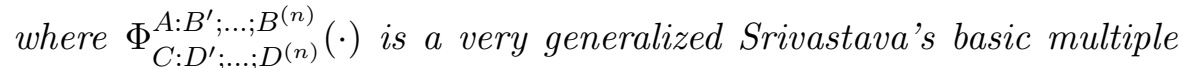
series (see [10, p. 350, Eq. (284)]) defined by

$(3.4)$

$$
\begin{aligned}
& \Phi_{C: D^{\prime}, \ldots ; D^{(n)}}^{A: B^{\prime} ; \ldots, B^{(n)}}\left(\left[\begin{array}{l}
{\left[(a): \theta^{\prime}, \ldots, \theta^{(n)}\right]:} \\
{\left[(c): \psi^{\prime}, \ldots, \psi^{(n)}\right]}
\end{array}\right]:\right. \\
& \left.\left.\left.\begin{array}{ll}
{\left[\left(b^{\prime}\right): \phi^{\prime}\right]} & \ldots ; \\
{\left[\left(d^{\prime}\right): \delta^{\prime}\right]} & \ldots ;
\end{array} \quad\left[\left(b^{(n)}\right): \phi^{(n)}\right] ; ; d^{(n)}\right): \delta^{(n)}\right] ; \quad q ; x_{1}, \ldots, x_{n}\right)
\end{aligned}
$$

$$
\begin{gathered}
\sum_{r_{1}, \ldots, r_{n}=0}^{\infty} \frac{\prod_{j=1}^{A}\left(a_{j} ; q\right)_{r_{1} \theta_{j}^{\prime}+\cdots+r_{n} \theta_{j}^{(n)}} \prod_{j=1}^{B^{\prime}}\left(b_{j}^{\prime} ; q\right)_{r_{1} \phi_{j}^{\prime}} \cdots \prod_{j=1}^{B^{(n)}\left(b_{j}^{(n)} ; q\right)_{r_{n} \phi_{j}^{(n)}}}}{\prod_{j=1}^{C}\left(c_{j} ; q\right)_{r_{1} \psi_{j}^{\prime}+\cdots+r_{n} \psi_{j}^{(n)}} \prod_{j=1}^{D^{\prime}}\left(d_{j}^{\prime} ; q\right)_{r_{1} \delta_{j}^{\prime}} \cdots \prod_{j=1}^{D^{(n)}\left(d_{j}^{(n)} ; q\right)_{r_{n} \delta_{j}^{(n)}}}} \\
\cdot \frac{x_{1}^{r_{1}}}{(q ; q)_{r_{1}}} \cdots \frac{x_{n}^{r_{n}}}{(q ; q)_{r_{n}}},
\end{gathered}
$$

where the arguments $x_{1}, \ldots, x_{n}$, the complex parameters

$$
\left\{\begin{array}{l}
a_{j}, j=1, \ldots, A ; b_{j}^{(k)}, j=1, \ldots, B_{j}^{(k)} ; \\
c_{j}, j=1, \ldots, C ; d_{j}^{(k)}, j=1, \ldots, D_{j}^{(k)} ; k=1, \ldots, n,
\end{array}\right.
$$

and the associated coefficients

$$
\left\{\begin{array}{l}
\theta_{j}^{(k)}, j=1, \ldots, A ; \phi_{j}^{(k)}, j=1, \ldots, B_{j}^{(k)} \\
\psi_{j}^{(k)}, j=1, \ldots, C ; \delta_{j}^{(k)}, j=1, \ldots, D_{j}^{(k)} ; k=1, \ldots, n,
\end{array}\right.
$$

are so constrained that the multiple series (3.4) converges.

Proof. We will prove Equation (3.1) only. The other two Equations (3.2) and (3.3) will be verified in a similar way. For convenience, let the left hand side of $(3.1)$ be denoted by $\mathcal{L}$. By using Equation (2.13), we obtain

$$
\begin{aligned}
\mathcal{L} & =\sum_{n=0}^{\infty}\left\{E_{q}\left(-\lambda_{1}\right) E_{q}\left(-\lambda_{2}\right) E_{q}\left(-\lambda_{3}\right)\right\}^{n} \\
& \cdot \sum_{r_{1}=0}^{n} \sum_{r_{2}=0}^{n-r_{1}} \sum_{r_{3}=0}^{n-r_{1}-r_{2}} \frac{q^{-\left({ }^{\left(r_{1}+r_{2}+r_{3}\right.}\right)}\left(q^{-n} ; q\right)_{r_{1}+r_{2}+r_{3}}\left(q^{-x_{1}} ; q\right)_{r_{1}}\left(q^{-x_{2}} ; q\right)_{r_{2}}\left(q^{-x_{3}} ; q\right)_{r_{3}}}{(q ; q)_{r_{1}+r_{2}+r_{3}}(q ; q)_{r_{1}}(q ; q)_{r_{2}}(q ; q)_{r_{3}}} \\
& \cdot\left\{q^{n}\left(1-e_{q}\left(\lambda_{1}\right)\right)\right\}^{r_{1}}\left\{q^{n}\left(1-e_{q}\left(\lambda_{2}\right)\right)\right\}^{r_{2}}\left\{q^{n}\left(1-e_{q}\left(\lambda_{3}\right)\right)\right\}^{r_{3}} t^{n}
\end{aligned}
$$

Employing a formal manipulation of double series (see [1]):

$$
\sum_{n=0}^{\infty} \sum_{l=0}^{n} A(l, n)=\sum_{n=0}^{\infty} \sum_{l=0}^{\infty} A(l, n+l),
$$


we have

$$
\begin{aligned}
\mathcal{L} & =\sum_{n=0}^{\infty} \sum_{r_{1}=0}^{\infty}\left\{E_{q}\left(-\lambda_{1}\right) E_{q}\left(-\lambda_{2}\right) E_{q}\left(-\lambda_{3}\right)\right\}^{n+r_{1}} \\
& \cdot \sum_{r_{2}=0}^{n} \sum_{r_{3}=0}^{n-r_{2}} \frac{q^{-\left({ }^{r_{1}+r_{2}+r_{3}}\right)}\left(q^{-n-r_{1}} ; q\right)_{r_{1}+r_{2}+r_{3}}\left(q^{-x_{1}} ; q\right)_{r_{1}}\left(q^{-x_{2}} ; q\right)_{r_{2}}\left(q^{-x_{3}} ; q\right)_{r_{3}}}{(q ; q)_{r_{1}+r_{2}+r_{3}}(q ; q)_{r_{1}}(q ; q)_{r_{2}}(q ; q)_{r_{3}}} \\
& \cdot\left\{q^{n+r_{1}}\left(1-e_{q}\left(\lambda_{1}\right)\right)\right\}^{r_{1}}\left\{q^{n+r_{1}}\left(1-e_{q}\left(\lambda_{2}\right)\right)\right\}^{r_{2}}\left\{q^{n+r_{1}}\left(1-e_{q}\left(\lambda_{3}\right)\right)\right\}^{r_{3}} t^{n+r_{1}} .
\end{aligned}
$$

By making a repeated use of (3.7), we get

$$
\begin{aligned}
\mathcal{L} & =\sum_{n=0}^{\infty} \sum_{r_{1}=0}^{\infty} \sum_{r_{2}=0}^{\infty} \sum_{r_{3}=0}^{\infty}\left\{E_{q}\left(-\lambda_{1}\right) E_{q}\left(-\lambda_{2}\right) E_{q}\left(-\lambda_{3}\right)\right\}^{n+r_{1}+r_{2}+r_{3}} \\
& \cdot \frac{q^{-\left({ }^{r_{1}+r_{2}+r_{3}}\right)}\left(q^{-n-r_{1}-r_{2}-r_{3}} ; q\right)_{r_{1}+r_{2}+r_{3}}\left(q^{-x_{1}} ; q\right)_{r_{1}}\left(q^{-x_{2}} ; q\right)_{r_{2}}\left(q^{-x_{3}} ; q\right)_{r_{3}}}{(q ; q)_{r_{1}+r_{2}+r_{3}}(q ; q)_{r_{1}}(q ; q)_{r_{2}}(q ; q)_{r_{3}}} \\
& \cdot\left\{q^{n+r_{1}+r_{2}+r_{3}}\left(1-e_{q}\left(\lambda_{1}\right)\right)\right\}^{r_{1}}\left\{q^{n+r_{1}+r_{2}+r_{3}}\left(1-e_{q}\left(\lambda_{2}\right)\right)\right\}^{r_{2}} \\
& \cdot\left\{q^{n+r_{1}+r_{2}+r_{3}}\left(1-e_{q}\left(\lambda_{3}\right)\right)\right\}^{r_{3}} t^{n+r_{1}+r_{2}+r_{3}} .
\end{aligned}
$$

Considering Equations (1.12) and (1.13), we find (3.8)

$$
\begin{aligned}
& \left(q^{-n-r_{1}-r_{2}-r_{3}} ; q\right)_{r_{1}+r_{2}+r_{3}} \\
& =\frac{(q ; q)_{n+r_{1}+r_{2}+r_{3}}}{(q ; q)_{n}}(-1)^{r_{1}+r_{2}+r_{3}} q^{\left({ }^{\left({ }_{1}+r_{2}+r_{3}\right.}\right)-\left(n+r_{1}+r_{2}+r_{3}\right)\left(r_{1}+r_{2}+r_{3}\right)}
\end{aligned}
$$

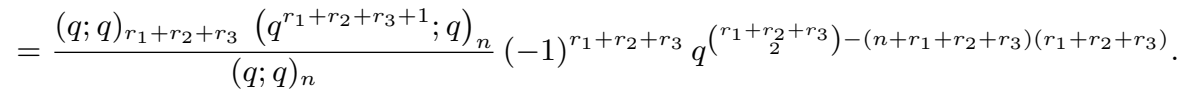

Applying the formula (3.8), we obtain

$$
\begin{aligned}
\mathcal{L}= & \sum_{r_{1}, r_{2}, r_{3}=0}^{\infty} \frac{\left(q^{-x_{1}} ; q\right)_{r_{1}}\left(q^{-x_{2}} ; q\right)_{r_{2}}\left(q^{-x_{3}} ; q\right)_{r_{3}}}{(q ; q)_{r_{1}}(q ; q)_{r_{2}}(q ; q)_{r_{3}}} \\
& \cdot\left\{t E_{q}\left(-\lambda_{1}\right) E_{q}\left(-\lambda_{2}\right) E_{q}\left(-\lambda_{3}\right)\left(1-e_{q}\left(\lambda_{1}\right)\right)\right\}^{r_{1}} \\
& \cdot\left\{t E_{q}\left(-\lambda_{1}\right) E_{q}\left(-\lambda_{2}\right) E_{q}\left(-\lambda_{3}\right)\left(1-e_{q}\left(\lambda_{2}\right)\right)\right\}^{r_{2}} \\
& \cdot\left\{t E_{q}\left(-\lambda_{1}\right) E_{q}\left(-\lambda_{2}\right) E_{q}\left(-\lambda_{3}\right)\left(1-e_{q}\left(\lambda_{3}\right)\right)\right\}^{r_{3}} \\
& \cdot \sum_{n=0}^{\infty} \frac{\left(q^{r_{1}+r_{2}+r_{3}+1} ; q\right)_{n}}{(q ; q)_{n}}\left\{t E_{q}\left(-\lambda_{1}\right) E_{q}\left(-\lambda_{2}\right) E_{q}\left(-\lambda_{3}\right)\right\}^{n}
\end{aligned}
$$


Using the $q$-binomial theorem (1.25) for the last summation and the identity (1.10), we find

$$
\begin{aligned}
& \sum_{n=0}^{\infty} \frac{\left(q^{r_{1}+r_{2}+r_{3}+1} ; q\right)_{n}}{(q ; q)_{n}}\left\{t E_{q}\left(-\lambda_{1}\right) E_{q}\left(-\lambda_{2}\right) E_{q}\left(-\lambda_{3}\right)\right\}^{n} \\
(3.10) & =\frac{\left(q^{r_{1}+r_{2}+r_{3}+1} t E_{q}\left(-\lambda_{1}\right) E_{q}\left(-\lambda_{2}\right) E_{q}\left(-\lambda_{3}\right) ; q\right)_{\infty}}{\left(t E_{q}\left(-\lambda_{1}\right) E_{q}\left(-\lambda_{2}\right) E_{q}\left(-\lambda_{3}\right) ; q\right)_{\infty}} \\
& =\frac{\left(q t E_{q}\left(-\lambda_{1}\right) E_{q}\left(-\lambda_{2}\right) E_{q}\left(-\lambda_{3}\right) ; q\right)_{\infty}}{\left(q t E_{q}\left(-\lambda_{1}\right) E_{q}\left(-\lambda_{2}\right) E_{q}\left(-\lambda_{3}\right) ; q\right)_{r_{1}+r_{2}+r_{3}}\left(t E_{q}\left(-\lambda_{1}\right) E_{q}\left(-\lambda_{2}\right) E_{q}\left(-\lambda_{3}\right) ; q\right)_{\infty}} .
\end{aligned}
$$

Now, substituting (3.10) for the last summation in (3.9) and using the identity (1.31), we obtain

$$
\begin{aligned}
\mathcal{L}= & \frac{\left(q t E_{q}\left(-\lambda_{1}\right) E_{q}\left(-\lambda_{2}\right) E_{q}\left(-\lambda_{3}\right) ; q\right)_{\infty}}{\left(t E_{q}\left(-\lambda_{1}\right) E_{q}\left(-\lambda_{2}\right) E_{q}\left(-\lambda_{3}\right) ; q\right)_{\infty}} \\
& \cdot \sum_{r_{1}, r_{2}, r_{3}=0}^{\infty} \frac{\left(q^{-x_{1}} ; q\right)_{r_{1}}\left(q^{-x_{2}} ; q\right)_{r_{2}}\left(q^{-x_{3}} ; q\right)_{r_{3}}}{\left(q t E_{q}\left(-\lambda_{1}\right) E_{q}\left(-\lambda_{2}\right) E_{q}\left(-\lambda_{3}\right) ; q\right)_{r_{1}+r_{2}+r_{3}}} \\
& \cdot \frac{\left\{t E_{q}\left(-\lambda_{2}\right) E_{q}\left(-\lambda_{3}\right)\left(1-E_{q}\left(-\lambda_{1}\right)\right)\right\}^{r_{1}}}{(q ; q)_{r_{1}}} \frac{\left\{t E_{q}\left(-\lambda_{1}\right) E_{q}\left(-\lambda_{3}\right)\left(1-E_{q}\left(-\lambda_{2}\right)\right)\right\}^{r_{2}}}{(q ; q)_{r_{2}}} \\
& \cdot \frac{\left\{t E_{q}\left(-\lambda_{1}\right) E_{q}\left(-\lambda_{2}\right)\left(1-E_{q}\left(-\lambda_{3}\right)\right)\right\}^{r_{3}}}{(q ; q)_{r_{3}}}
\end{aligned}
$$

Finally, by considering the generalized multiple series in (3.4), the last resulting triple series (3.11) corresponds with Equation (3.1). The other two Equations (3.2) and (3.3) will be proved by a similar argument. This completes the proof of Theorem.

\section{References}

[1] J. Choi, Notes on formal manipulations of double series, Commun. Korean Math. Soc. 18 (2003), 781-789.

[2] J. Choi, A generalization of Gottlieb polynomials in several variables, Appl. Math. Lett. 25 (2012), 43-46; DOI: 10.1016/j.aml.2011.07.006.

[3] J. Choi, $q$-Extension of a generalization of Gottlieb polynomials in two variables, J. Chungcheong Math. Soc. 25 (2012), 253-265.

[4] G. Gasper and M. Rahman, Basic Hypergeometric Series (with a Foreword by Richard Askey), Encyclopedia of Mathematics and Its Applications, Vol. 35, Cambridge University Press, Cambridge, New York, Port Chester, Melbourne and Sydney, 1990; Second edition, Encyclopedia of Mathematics and Its Applications, Vol. 96, Cambridge University Press, Cambridge, London and New York, 2004.

[5] M. J. Gottlieb, Concerning some polynomials orthogonal on a finite or enumerable set of points, Amer. J. Math. 60(2) (1938), 453-458.

[6] M. A. Khan and M. Akhlaq, Some new generating functions for Gottlieb polynomials of several variables, Internat. Trans. Appl. Sci. 1(4) (2009), 567-570. 
[7] M. A. Khan and M. Asif, A note on generating functions of $q$-Gottlieb polynomials, Commun. Korean Math. Soc. (2011), Accepted for publication.

[8] G. Lauricella, Sulle funzioni ipergeometriche a piú variabili, Rend. Circ. Mat. Palermo 7 (1893), 111-158.

[9] E. D. Rainville, Special Functions, Macmillan Company, New York, 1960; Reprinted by Chelsea Publishing Company, Bronx, New York, 1971.

[10] H. M. Srivastava and P. W. Karlsson, Multiple Gaussian Hypergeometric Series, Halsted Press (Ellis Horwood Limited, Chichester), John Wiley and Sons, New York, Chichester, Brisbane, and Toronto, 1985.

[11] H. M. Srivastava and H. L. Manocha, A Treatise on Generating Functions, Halsted Press (Ellis Horwood Limited, Chichester), John Wiley and Sons, New York, Chichester, Brisbane, and Toronto, 1984.

Junesang Choi

Department of Mathematics, Dongguk University, Gyeongju 780-714, Korea.

E-mail: junesang@mail.dongguk.ac.kr 